\title{
Collective Scattering of Light on Gold Nanospheres Dispersed in Diethylene Glycol Microdroplet
}

\author{
M. Kolwas, K. Kolwas, D. Jakubczyk and G. Derkachov \\ Institute of Physics, Polish Academy of Sciences, Aleja Lotnikow 32/46, PL-02668 Warsaw, Poland
}

(Received December 15, 2016; in final form January 18, 2017)

\begin{abstract}
Light scattering on a freely suspended, evaporating droplet of suspension of gold nanospheres (125 nm radius) in diethylene glycol is studied, revealing both local and nonlocal properties of the system. When the average distance between the nanospheres at the droplet surface matches the wavelength of light, a (broad) maximum, associated with the collective scattering on them, is observed. The number of nanospheres at the droplet surface can be found then. A fine modulation of the maximum is associated with the whispering gallery modes of the composite droplet. The Fano profile identified in the modulation is interpreted as an interference of collective scattering and whispering gallery modes. The ultra narrow enhancement-and-quench structure recognizable in the modulation is interpreted as associated with the creation and destruction of collective oscillation of plasmons of individual nanospheres. It is proposed that this phenomenon can be perceived as whispering gallery modes-assisted creation and destruction of a global plasmon.
\end{abstract}

DOI: 10.12693/APhysPolA.131.288

PACS/topics: whispering gallery modes, Fano profile, plasmon, micro-cavities, collective scattering, droplet with inclusions

\section{Introduction}

Light scattering is often influenced by various resonances resulting from phase and frequency matching in its interaction with the scatterer. For example, the total intensity, as well as the intensity distribution of light scattered by a dielectric sphere shows a rich variety of structural resonances [1-5]. A particular class of these resonances, associated with the standing waves at the spherical surface, are referred to as whispering gallery modes (WGMs, see e.g. $[6,7])$. For inhomogeneous or composite droplets, additional structural resonances resulting from the light wavelength matching to the characteristic lengths of the droplet internal structure (inhomogeneity) can also manifest. These additional structural resonances can be modified by the fundamental ones. For example, in case of droplets of dielectric suspensions, WGMs (of the entire droplet) can interact with the scattering on the surface inclusions [8]. The resonance conditions can enhance or decrease scattering significantly. In case of a coincidence of resonances of different origin, a significant enhancement/decrease can be expected. Such enhancement of scattering was studied in a layered, metaldielectric structure of a sub-wavelength sphere $[9,10]$. We expect that similar phenomena can manifest also in other complex metal-dielectric systems.

In this paper we studied light scattering on an evaporating microdroplet of suspension of gold nanospheres (GNS) in diethylene glycol (DEG). During DEG evaporation, the concentration of GNS at the droplet surface increases significantly faster than in the volume. It is also expected that GNS distribution at the surface becomes fairly regular with growing concentration, due to the electrostatic repulsion. This induces changes of the surface properties from dielectric-like to metal-like, and allows for the Bragg-like scattering on a lattice of surface
GNS. The average distance between GNS favors certain WGMs (Bragg-like condition) and the arising scattering maximum can be perceived as the collective scattering (CS). In contrast to dielectric inclusions [8, 11], the high coupling between WGMs and scattering on the surface lattice of GNS can lead to synchronization of plasmons on individual inclusions. This can be perceived as the creation of a global plasmon [12].

The average distance between inclusions is controlled by their concentration and, in consequence, was scanned during the evaporation of the dispersion medium. Slow evaporation of DEG (slow droplet radius change) enabled a detailed study of various kinds of resonances as well as their interactions. We studied two slightly different systems: (A) a droplet with low initial concentration of GNS $(\approx 60$ GNS in a droplet of $\approx 16 \mu \mathrm{m}$ initial radius $)$ and (B) a droplet with $7.2 \times$ higher concentration of inclusions $(\approx 125$ GNS in a droplet of $\approx 10.6 \mu \mathrm{m}$ initial radius $)$. We identified and studied several phenomena:

1. manifestation of CS resonance, associated with the GNS at the surface,

2. modulation of CS maximum with WGMs and interference of WGMs associated with waves travelling in different media,

3. Fano interference of CS and WGM,

4. WGM-assisted synchronization of plasmons on individual GNS and formation of a global plasmon.

Some of the phenomena were observed in both systems, while the rest - only in one of them.

\section{Measurement concept and experimental setup}

The studied droplets of suspension were singly levitated in an electrodynamic quadrupole trap [13-16] built 
in our laboratory $[8,17,18]$. A proper combination of alternating (AC) and static (DC) electric fields, in (nearly) quadrupolar configuration, enables constraining a charged particle to a very small volume, in practice to a point. The trap was kept in a small $\left(\approx 10 \mathrm{~cm}^{3}\right)$ thermostatic chamber with dry nitrogen at atmospheric pressure and $25^{\circ} \mathrm{C}$. Single droplets were injected into the trap with the droplet-on-demand injector (built in our lab as well; similar to e.g. $[19,20])$ kept at the chamber temperature. The droplets were charged by charge separation in the (external) field of the trap, on emerging from the injector nozzle. Thus, the sign and, to some extent, the value of the charge was determined by the injection timing versus the phase of the trapping AC field. Our setup allows stable trapping of droplets of radius in the range from $\approx 35 \mu \mathrm{m}$ to $\approx 0.5 \mu \mathrm{m}$. The average initial radius was several $\mu \mathrm{m}$.

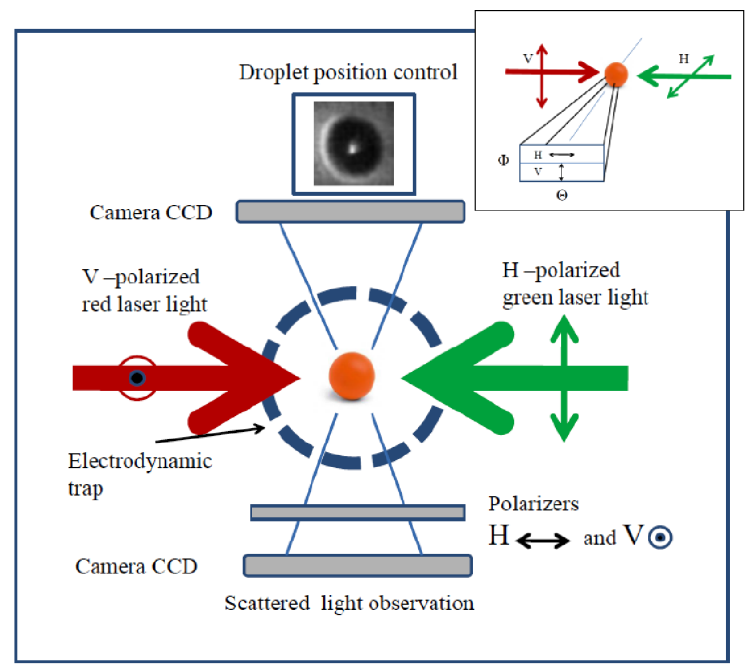

Fig. 1. Measurement concept (top view in main part, side view in inset). Main part: top - droplet vertical position stabilization system using in-focus image of the droplet. Middle - arrangement of the illumination and observation channels relative to the trap. Bottom - out-of-focus image (scattered light intensity) acquisition. Inset - the details of the observation channel geometry.

For sample preparation we used diethylene glycol (DEG), 99.99 GC area \% (BioUltra, Fluka) and colloid suspension of GNS of $125 \mathrm{~nm}$ radius in water, $3.6 \times 10^{8}$ particles $/ \mathrm{ml}$ (BBInternational), which we mixed in 1:4 volume proportion and de-gassed and dried under vacuum. Thus we expect initial concentration of $\approx 1.4 \times 10^{9} \mathrm{GNS} / \mathrm{ml}$ of DEG.

The concept of the experiment is shown schematically in Fig. 1. Two coaxial, counter propagating laser light beams were used simultaneously for (static, elastic) scattering on the droplet: green $(532 \mathrm{~nm}, 5.5 \mathrm{~mW})$ horizontally polarized (H-polarized) and red (658 nm, $10 \mathrm{~mW}$ ) vertically polarized ( $\mathrm{V}$-polarized) in respect of the scattering plane.
Two linear polarizers were used in the detection channel: H-polarizer (upper half of the field of view) and the $\mathrm{V}$-polarizer (the lower half). Using a color camera allowed us to separate the scattered light spectrally and attribute it to appropriate incident beam polarization. We recorded temporal evolution of the scattered light intensity angular distribution. In post-processing, the spherical and chromatic aberrations introduced by the lens system were corrected and the distributions were integrated in appropriate detection channels over scattering angles $\Theta=90 \pm 16.24^{\circ}$ (azimuth) and $\Phi=0 \pm 5.33^{\circ}$ (elevation). The scattered light intensities of the same polarization as the incident are denoted as $I_{V V}(\Phi, \Theta ; t)$ (red light) and $I_{H H}(\Phi, \Theta ; t)$ (green light). The, so called, cross-polarized scattered light intensities are denoted as $I_{V H}(\Phi, \Theta ; t)$ - red and $I_{H V}(\Phi, \Theta ; t)$ - green. For simplicity we denote intensity integrated over both $\Phi$ and $\Theta$ angles as $I$, while integrated over $\Phi$ only, as $I(\Theta)$.

The droplet radius evolution $R(t)$ was determined by analyzing (mostly with the look-up table method in the framework of the Mie theory [21]) the scatterograms sequence $\left(I_{V V}(\Theta, t)\right.$ and $\left.I_{H H}(\Theta, t)\right)$ and by weighting with the help of the DC field of the trap [18]. The combination of these methods yields $R(t)$ very accurately for a wide range of suspension concentrations.

\section{Scattering of light on a droplet with low concentration of GNS}

In this section, we study the light scattering on the droplet with low initial GNS concentration. The temporal evolution of the scattered light intensities, that we consider, is shown in Fig. 2. These are: $I_{V V}$ (red light, polarization retained), $I_{H H}$ (green light, polarization retained), $I_{V H}$ (red, cross-polarized light) and $I_{H V}$ (green, cross-polarized light). Fast oscillations of $I_{V V}$ and $I_{H H}$ signals - shown magnified in inset in Fig. 2 - are due to WGM of the droplet.

The cross-polarized intensities: $I_{V H}$ and $I_{H V}$ are small but substantial. Since scattering on a homogeneous spherical droplet practically does not lead to light depolarization near the equatorial plane, the $I_{V H}$ and $I_{H V}$ signals shall be associated with GNS. The electromagnetic (EM) field inside the droplet does not propagate along the incident light beam direction and the wave vector is only a local property. Scattering of such field on GNS (ensemble) in general does not retain the polarization of the incident wave and the cross-polarized (depolarized) scattered light appears.

During the suspension droplet evaporation, the concentration of the dispersed phase is growing nearly exclusively at the surface, where it accumulates from the evaporated dispersion medium volume. However, in case of a GNS suspension illuminated with a laser beam, the GNS interaction with EM field (photophoretic force, optical gradient force) should also be considered [22, 23]. For a droplet with low initial concentration of GNS, shown in Fig. 2, both mechanisms influencing their distribution 


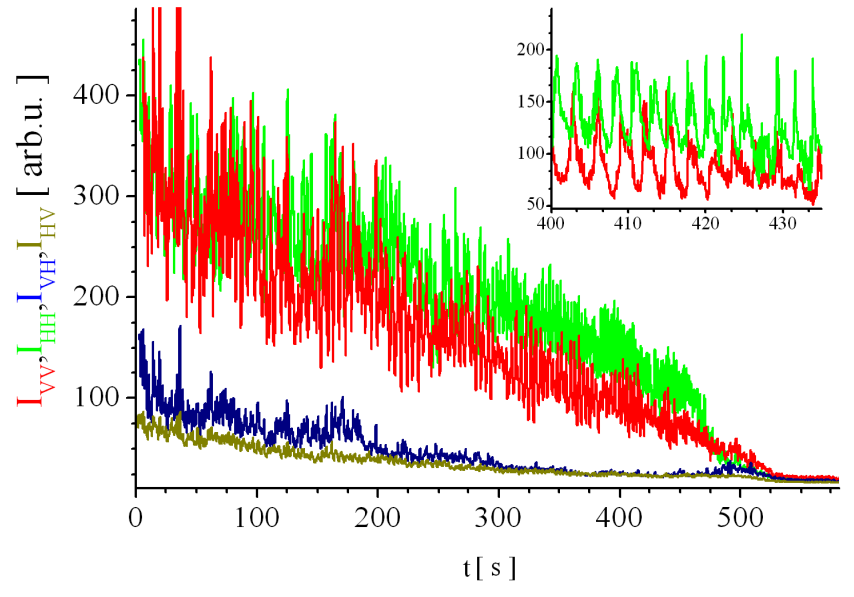

Fig. 2. The experimentally obtained temporal evolution of the scattered light intensities for a droplet with low initial GNS concentration: red line $-I_{V V}$ (red light, polarization retained), green line $-I_{H H}$ (green light, polarization retained), blue line $-I_{V H}$ (red, cross-polarized light) and dark yellow $-I_{H V}$ (green, cross-polarized light). Fast oscillations of $I_{V V}$ and $I_{H H}$ signals - shown magnified in inset - are due to WGM of the droplet.

should be considered. We expect that interaction of GNS with the droplet internal light field may be at play at initial stages of evolution, until $460 \mathrm{~s}$. The analysis of the influence, which such interaction exerts upon the observed scattered light intensities and the structure of the resonances is beyond the scope of this work. However, in the discussed case, the CS resonance appears at the later stages of evaporation, when the interaction becomes much smaller.

\subsection{Manifestation of the collective scattering resonance, associated with the surface GNS}

The collective scattering resonance manifests when the average distance between GNS matches the wavelength of light (compare [8] for dielectric inclusions). In order to study the phenomenon of collective scattering on GNS, first we analyzed the evolution of $I_{V V}$ and $I_{V H}$ intensities. We collated $I(t)$ with the corresponding $I(\Theta, t)$ maps (Fig. 3), and compared the temporal evolution of WGMs with the spatial distribution evolution of scattered light - in particular with the interference fringes manifestation (typical for scattering on uniform droplet, see e.g. [18]). The interference fringes are visible as vertical white dashed lines in $I_{V V}(\Theta, t)$ map in Fig. 3. For $t<450 \mathrm{~s}$ they are visible with high contrast, indicating that the droplet (surface) is homogeneous. At the same time, WGMs seen in both $I_{V V}$ and $I_{H H}$ signals (see Fig. 2), are well pronounced and regular. For $t>450 \mathrm{~s}$ the interference fringes become diffused, and at $\approx 490 \mathrm{~s}$ they totally disappear as the droplet (surface) loses its homogeneity. Correspondingly, WGMs become less regular and pronounced. Then, the maximum associated with CS resonance is plainly seen in $I_{V H}$ signal at $\approx 500 \mathrm{~s}$ of evolution.

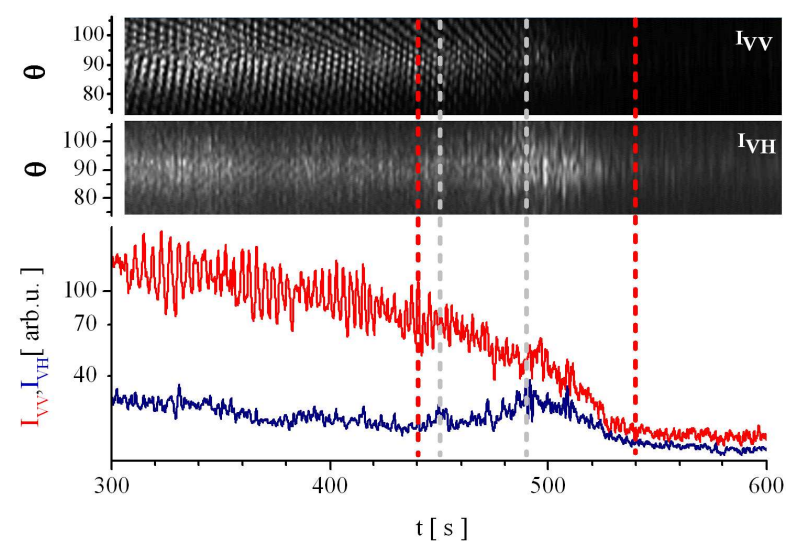

Fig. 3. Upper parts: the temporal evolution of $I_{V V}(\Theta, t)$ and $I_{V H}(\Theta, \mathrm{t})$ angular intensity distributions (brightness-coded maps). Lower part: the temporal evolution of $I_{V V}$ (red, upper line) and $I_{V H}$ (blue, lower line) intensities (integrated over $\Theta$ ). High contrast interference fringes can be seen on $I_{V V}(\Theta, t)$ map until $\approx 450 \mathrm{~s}$ (left gray vertical dashed line). After $450 \mathrm{~s}$ the interference fringes become diffused, and at $\approx 490 \mathrm{~s}$ (right gray vertical dashed line) the regularity of pattern is totally lost. Between $\approx 440 \mathrm{~s}$ and $\approx 540 \mathrm{~s}$ (red vertical dashed lines) a well pronounced (modulated) maximum, associated with CS resonance, can be seen in both $I_{V H}(\Theta, t)$ and $I_{V H}(t)$.

In order to study the CS resonance in detail we have developed the following procedure: First, we extract the main evolution trends versus the droplet radius of intensity of light scattered by GNS independently: $I_{H V}^{0}(R)$ and $I_{V H}^{0}(R)$. This can be done, for instance, by fitting polynomials of lowest possible order (we found 3rd to be sufficient) to $I_{H V}(R)$ and $I_{V H}(R)$ with CS region (420$540 \mathrm{~s}$ ) excluded.

Then, CS resonance can be emphasized by constructing the ratios $I_{H V}^{N}=I_{H V} / I_{H V}^{0}$ and $I_{V H}^{N}=$ $I_{V H} / I_{V H}^{0}$ and presenting them versus the droplet radius (see Fig. 4). For each signal, a compound profile of the maximum is revealed then. It can be fairly well reproduced with a linear combination of two Gaussian profiles. We denominate the component maxima manifesting for smaller droplet radii as $L$, and for larger droplet radii as $R$. Both maxima exhibit fine modulation associated with the interaction between scattering on GNS and WGMs.

\subsection{Modulation of CS maximum with WGMs and} interference of WGMs associated with waves travelling in different media

In this section we show that $R$ and $L$ maxima are associated with the Bragg-like scattering of waves travelling below and above the (average) droplet surface and that the interference between WGMs associated with these waves can be observed as beating. 


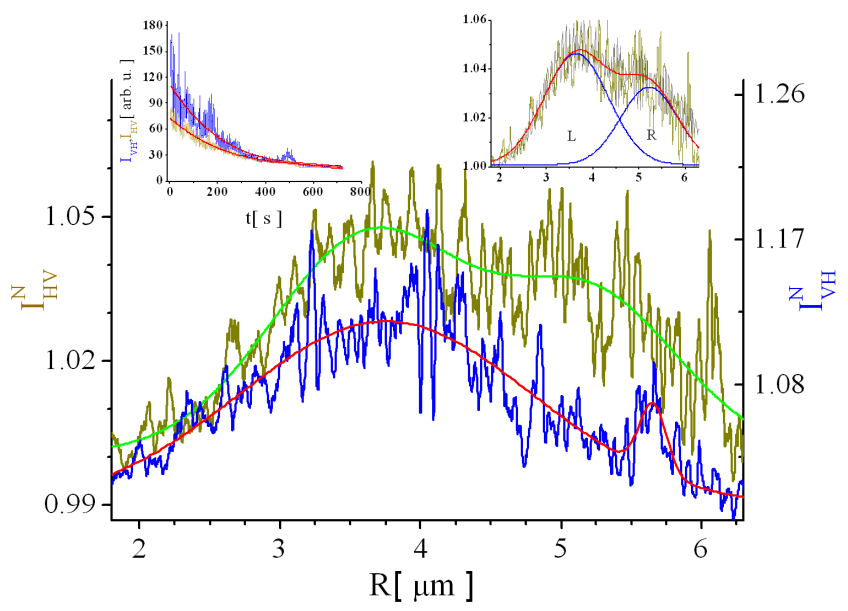

Fig. 4. Main part: the manifestation of CS resonance in cross-polarized signals $I_{H V}^{N}(R)$ and $I_{V H}^{N}(R)$. The CS maximum is decomposed into two Gaussian profiles with centers at $R_{L}=3.7 \mu \mathrm{m}$ and $R_{R}=5.2 \mu \mathrm{m}$ for the green light and at $R_{L}=3.75 \mu \mathrm{m}$ and $R_{R}=5.65 \mu \mathrm{m}$ for the red light. Modulation of CS maxima with beating WGMs can be seen. Right inset: fitting of CS maximum with two Gaussian profiles. Left inset: fitting of $I_{H V}$ and $I_{V H}$ with 3 rd order polynomials.

The $R$ maximum for the green light, with the center at $R_{R}=5.2 \pm 0.1 \mu \mathrm{m}$, is fairly broad and seems modulated with two WGMs of slightly different periods (vs. the droplet radius): $58 \pm 3.5$ and $66 \pm 3.5 \mathrm{~nm}$, respectively. The WGM period calculated as $\delta R=\lambda / 2 \pi m$ for propagation in DEG $(m=1.45)$ is $58.5 \mathrm{~nm}$, while for air $(m=1)$ it is $85 \mathrm{~nm}$. This suggests that the first of WGM is localized below the (average) surface, while the second - rather unexpectedly above (Fig. 5).

The $L$ maximum for the green light, with the center at $R_{L}=3.7 \pm 0.1 \mu \mathrm{m}$, is also broad, and is modulated with a single WGM of $83.7 \pm 3.5 \mathrm{~nm}$ period. This again corresponds to theoretical $84.7 \mathrm{~nm}$ for propagation in air.

Furthermore, the ratio of the maxima positions $R_{R} / R_{L}=5.2 / 3.7=1.405 \pm 0.065$ is within the error limit from the DEG and air refractive indices ratio (1.45:1).

Using the resonance condition either for $R$ or for $L$ maximum for the green light enables estimation of the number of (surface) GNS participating in the scattering as $72 \pm 10$.

The $L$ maximum for the red light, at $R_{L}=3.75 \pm 0.15$, is very well pronounced and the $R$ maximum, at $R_{R}=$ $5.65 \pm 0.05$, is significantly smaller but narrower. The ratio $R_{R} / R_{L}=1.51 \pm 0.07$ is again close to the ratio of DEG and air refractive indices. Again, the resonance condition enables estimation of the number of (surface) GNS participating in the scattering. For the red light we get $55 \pm 8$ inclusions. This is within the error limit from the result for the green light. The average between the two results allows a better estimation of $64 \pm 9$ inclusions.

The $L$ maximum is again modulated with two beating WGMs, with periods of $81.6 \pm 4$ and $100 \pm 4 \mathrm{~nm}$. The

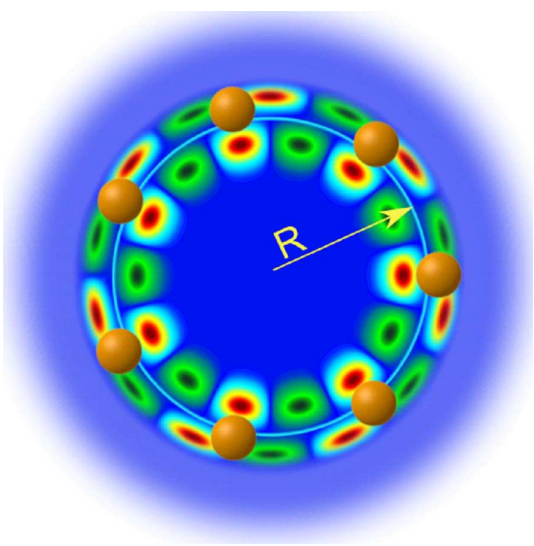

Fig. 5. The concept of matching of WGM (simplified local light field intensity color-coded) to the GNS lattice (golden spheres). The standing wave length inside and outside of the (average) droplet surface $(n \lambda / m=2 \pi)$ generally does not match the average distance between the spheres simultaneously.

calculated $\delta R$ is respectively $72 \mathrm{~nm}$ in DEG and $104 \mathrm{~nm}$ in air. This fairly good agreement again supports the hypothesis of WGMs below and above the droplet surface.

This suggests that the $R$ and $L$ maxima correspond to the Bragg-like scattering on GNS immersed in DEG and in air, respectively.

\section{Scattering of light on a droplet with higher initial concentration of GNS}

\subsection{Strong influence of CS on the scattered light intensities}

In case of a suspension droplet with the low initial GNS concentration, the intensity maxima associated with CS were clearly seen but rather small in comparison to the scattered light intensity at earlier stages of the evolution. In contrast, for a droplet with higher initial GNS concentration, the CS phenomenon should dominate and the transition from the scattering on the entire droplet to domination of scattering on GNS would not be visible. Indeed, broad maxima, with a fine and very deep modulation, visible directly for the red light, not only in cross-polarized $I_{V H}$ but even in $I_{V V}$ signal, can be associated with the CS resonance. In Fig. 6, they can be seen for the droplet radii between 9 and $7.5 \mu \mathrm{m}$. It is hardly possible to recognize CS in green scattered light, since the absorption of green light in GNS with $125 \mathrm{~nm}$ radius is $\approx 4 \times$ higher than of red. CS can be expected to manifest more strongly for a larger droplet (higher number of GNS) then.

The position of the maximum of the red scattered light intensity (at $8.5 \mu \mathrm{m}$ ) was used to estimate the number of inclusion as $\approx 125 \pm 10$. This is twice the number found for the low-concentration case. Due to a high number of inclusions the cross-polarized signal $I_{V H}$ (blue line in Fig. 6) is only slightly lower than $I_{V V}$ (red line). 


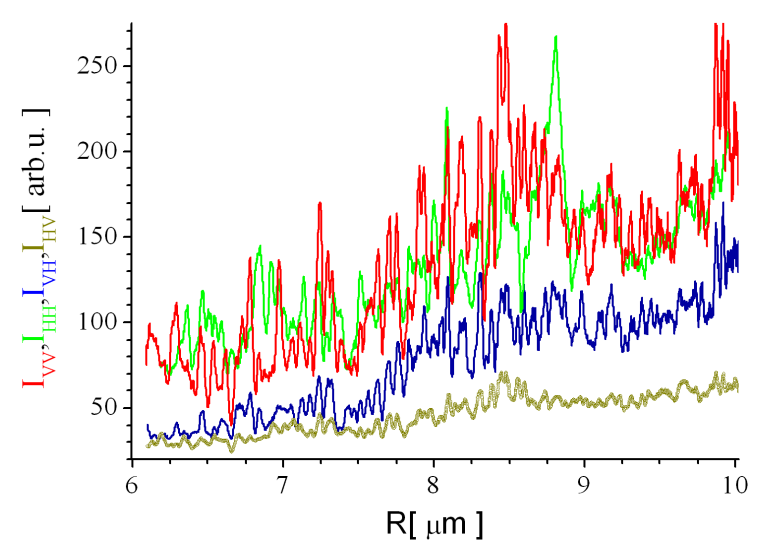

Fig. 6. The experimentally obtained temporal evolution of the scattered light intensities for the case of higher initial concentration of GNS (compare Fig. 2): red line $-I_{V V}$, green line $-I_{H H}$, blue line $-I_{V H}$ and dark yellow $-I_{H V}$.

\subsection{Interaction of CS resonance with WGMs - Fano profile and beating of WGMs standing in different media}

In order to study the details of CS we constructed $I_{V V}^{N}=I_{V V} / I_{V V}^{0}$ and $I_{V H}^{N}=I_{V H} / I_{V H}^{0}$ ratios and plotted them versus $R$ (see Fig. 7; it is worth noticing that $I_{V V}^{N}$ exceeds 2). The CS maxima in both signals can again be approximated with broad Gaussian profiles. However, it must be kept in mind that $I_{V V}^{N}$ signal still carries information on both scattering on GNS and on the entire droplet. The interference of these two modes of scattering results in a Fano profile, which can be observed near the center of $I_{V V}^{N}(R)$ maximum (compare: scattering of light on dielectric inclusions forming a surface layer in a droplet [11]). Simultaneously, an interference of two WGMs standing below and above the droplet surface can be identified, as the characteristic beating, in cross-polarized $I_{V H}^{N}$ signal (Fig. 7).

\subsection{WGM-assisted synchronization of plasmons on individual GNS and formation of a global plasmon}

It is worth noticing that a WGM minimum can entirely quench the CS maximum (e.g. at $R=8.34 \mu \mathrm{m}$ in Fig. $7, T_{2}=97.93 \mathrm{~s}$ in Fig. 8). GNS scatter independently at WGMs minima, while at WGMs maxima (e.g. $R=8.37 \mu \mathrm{m}$ in Fig. $7, t=100.7 \mathrm{~s}$ in Fig. 8) they scatter collectively. This phenomenon of very strong coupling/decoupling between CS and WGMs manifests as dark and bright super-narrow (vertical dashed) lines (less than $40 \mathrm{~nm}$ wide) in $I_{V V}(\Theta, t)$ map in Fig. 8. Equivalently, it manifests as the revival/collapse of the interference fringes seen in $I(\Phi, \Theta)$ (movie frames in Fig. 8). When light is scattered on an ideally homogeneous droplet, only the interference fringes can be seen (frame $t_{0}$ in Fig. 8: no cross-polarized $I_{V H}$ at the very beginning of the droplet evolution). For light independently scattered on GNS (out-off WGM resonance) only speckles can be seen (in both $I_{V V}(\Phi, \Theta)$ and $I_{V H}(\Phi, \Theta)$, frame

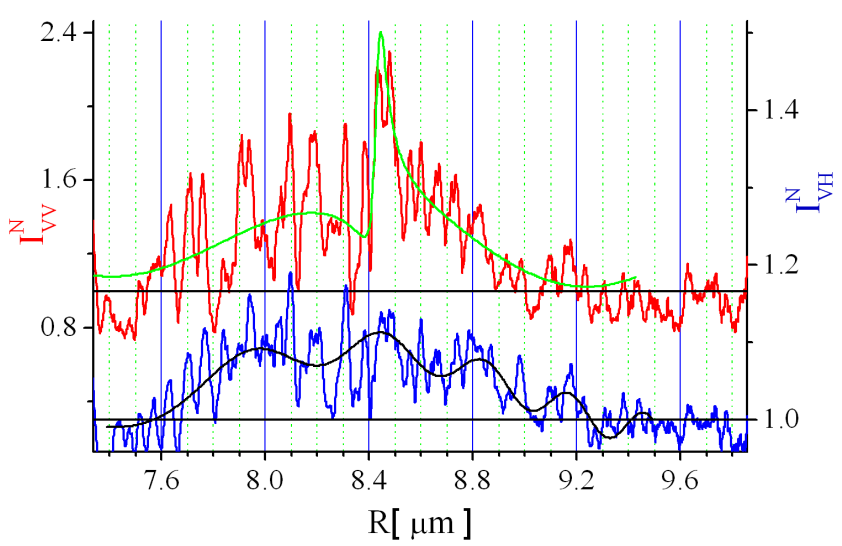

Fig. 7. The broad maxima associated with CS resonance seen in $I_{V V}^{N}(R)$ (red, upper line) and $I_{V H}^{N}(R)$ (blue, lower line). Complete quenching of CS maximum by WGM minimum can be seen in $I_{V V}^{N}$ signal e.g. for $R=8.34 \mu \mathrm{m}$. The interference (beating) of WGMs standing below and above the surface can be seen in $I_{V H}^{N}$ signal as the modulation of a Gaussian profile (black line). A Fano profile can be seen in $I_{V V}^{N}$ signal near the center of a Gaussian profile (green line).

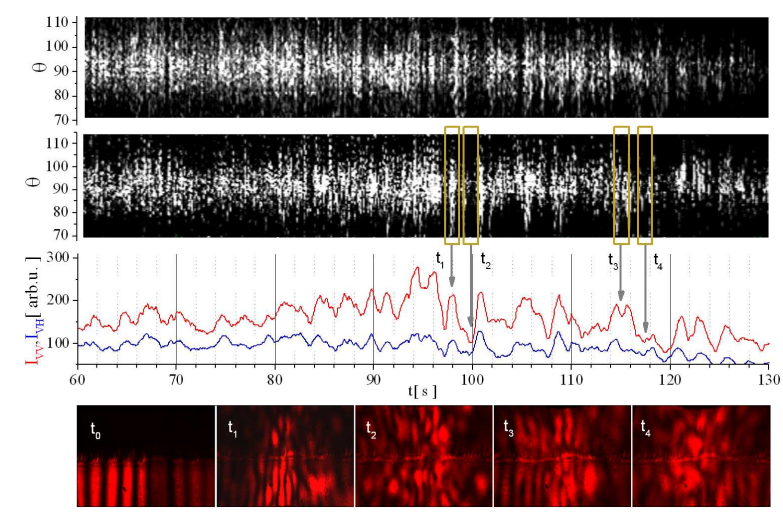

Fig. 8. Top and second from the top parts: $I_{V V}(\Theta, t)$ and $I_{V H}(\Theta, t)$ maps, respectively. Third from the top part: the temporal evolution of the scattered light intensities $I_{V V}(t)$ and $I_{V H}(t)$. Very narrow WGM maxima can be seen as a modulation of the very broad CS maximum. Bottom part: the observed $I(\Theta, \varphi)$ (selected movie frames; see Sect. 2) corresponding to the temporal positions indicated in other parts (gray arrows, yellow rectangles); $t_{0}$ corresponds to the beginning of the droplet evaporation.

$t_{4}$ in Fig. 8). However, for WGM maxima coinciding with CS maximum, the interference fringes partly revive (frames $t_{1}$ and $t_{3}$ in Fig. 8). They sometimes seem to appear in cross-polarized light as well (frame $t_{3}$ in Fig. 8). This would signify that the polarization of the associated spherical cavity mode of entire droplet is skewed then. Since scattering of red light on $125 \mathrm{~nm}$ radius GNS has plasmonic nature, the WGM-CS coupling can be perceived as synchronization of plasmons on individual GNS and formation of a global plasmon. The phenomenon is 
similar to the atomic/molecular dipole synchronization leading to creation of a macroscopic dipole [24].

\section{Conclusions}

An evaporating microdroplet of GNS suspension was used as a study of meta-material object with variable optical properties. The properties under study were modified due to the evaporation-driven changes of the GNS distribution in the droplet. The maximum of the distribution formed at the droplet surface. Several scattering phenomena associated with different resonant conditions corresponding to specific GNS distributions were also observed and identified. The resonant conditions for light scattering on a droplet of suspension are twofold. There are modes of the spherical resonator (the composite droplet as a whole) and resonances arising due to matching of the average distance between (surface) inclusions to the light wavelength. There are also very significant interactions between the two. During the droplet evaporation a variety of (consecutive) resonances was scanned. When the average distance between surface GNS was matching the wavelength of light, a broad maximum associated with the collective scattering (CS) on GNS was observed. A fine modulation of this maximum is associated with the whispering gallery modes (WGMs) of the composite droplet. WGMs associated with waves travelling below and above the (average) droplet surface can be recognized. The Fano profile identified in the modulation is interpreted as an interference of CS and WGMs. The ultra narrow enhancement-and-quench structure recognizable in the modulation is interpreted as associated with the creation and destruction of collective oscillation of individual GNS plasmons (WGM-assisted creation and destruction of a global plasmon).

Resonant phenomena are potential tools for precise measurements. We were able, for instance, to find the number of surface GNS with a fair accuracy. Engineering applications based on tuning the variable optical properties of the meta-material object seems also potentially feasible.

\section{Acknowledgments}

This work was supported by the National Science Center, Poland under grants number 2014/13/B/ST3/04414 and 2014/13/D/ST3/01882.

\section{References}

[1] M. Tona, Study on Spherical Microlasers Levitated in an Ion Trap, Kochi University, 2002.

[2] K.J. Vahala, Nature 424, 839 (2003).

[3] M.I. Mishchenko, J. Quant. Spectrosc. Radiat. Transf. 110, 1210 (2009).

[4] P. Chýlek, J.T. Kiehl, M.K.W. Ko, Phys. Rev. A 18, 2229 (1978)

[5] A.V. Kavokin, J.B. Jeremy, G. Malpuech, F.P. Laussy, Microcavities, OUP, Oxford 2008.

[6] A.B. Matsko, V.S. Ilchenko, IEEE J. Sel. Top. Quantum Electron. 12, 3 (2006).

[7] W. von Klitzing, R. Long, V.S. Ilchenko, J. Hare, V. Lefèvre-Seguin, New J. Phys. 3, 14.1 (2001).

[8] M. Kolwas, D. Jakubczyk, G. Derkachov, K. Kolwas, J. Quant. Spectrosc. Radiat. Transf. 131, 138 (2013).

[9] Z. Ruan, S. Fan, Phys. Rev. Lett. 105, 013901 (2010).

[10] Z. Ruan, S. Fan, Appl. Phys. Lett. 98, 043101 (2011).

[11] M. Kolwas, K. Kolwas, G. Derkachov, D. Jakubczyk, Phys. Chem. Chem. Phys. 17, 6881 (2015).

[12] S.K. Ghosh, T. Pal, Chem. Rev. 107, 4797 (2007).

[13] W. Paul, Rev. Mod. Phys. 62, 531 (1990).

[14] D.J. Berkeland, J.D. Miller, J.C. Bergquist, W.M. Itano, D.J. Wineland, J. Appl. Phys. 83, 5025 (1998).

[15] E.J. Davis, M.F. Buehler, T.L. Ward, Rev. Sci. Instrum. 61, 1281 (1990).

[16] S. Arnold, Rev. Sci. Instrum. 62, 3025 (1991).

[17] D. Jakubczyk, G. Derkachov, T. Do Duc, K. Kolwas, M. Kolwas, J. Phys. Chem. A 114, 3483 (2010).

[18] D. Jakubczyk, G. Derkachov, M. Kolwas, K. Kolwas, J. Quant. Spectrosc. Radiat. Transf. 126, 99 (2013).

[19] H. Ulmke, T. Wriedt, H. Lohner, K. Bauckhage, Precis. Eng. Nanotechnol. 2, 290 (1999).

[20] G. Brenn, Chem. Eng. Sci. 55, 5437 (2000).

[21] C.F. Bohren, D.P. Huffman, Absorption and Scattering of Light by Small Particles, Springer, Berlin 1983.

[22] S. Fardad, A. Salandrino, M. Heinrich, P. Zhang, Z. Chen, D.N. Christodoulides, Nano Lett. 14, 2498 (2014).

[23] R. El-Ganainy, D.N. Christodoulides, C. Rotschild, M. Segev, Opt. Express 15, 10207 (2007).

[24] R.H. Dicke, Phys. Rev. 93, 99 (1954). 